\title{
Human Detection and Tracking via Ultra-Wideband (UWB) Radar
}

\author{
SangHyun Chang, Michael Wolf, and Joel W. Burdick
}

\begin{abstract}
This paper presents an algorithm for human presence detection and tracking using an Ultra-Wideband (UWB) impulse-based mono-static radar. UWB radar can complement other human tracking technologies, as it works well in poor visibility conditions. UWB electromagnetic wave scattering from moving humans forms a complex returned signal structure which can be approximated to a specular multipath scattering model (SMPM). The key technical challenge is to simultaneously track multiple humans (and non-humans) using the complex scattered waveform observations. We develop a multiple-hypothesis tracking (MHT) framework that solves the complicated data association and tracking problem for an SMPM of moving objects/targets. Human presence detection utilizes SMPM signal features, which are tested in a classical likelihood ratio (LR) detector framework. The process of human detection and tracking is a combination of the MHT method and the LR human detector. We present experimental results in which a mono-static UWB radar tracks human and non-human targets, and detects human presence by discerning human from moving non-human objects.
\end{abstract}

\section{INTRODUCTION}

This paper introduces an algorithm for Ultra-Wideband (UWB) radar-based human presence detection and tracking. Our particular motivation is the problem of outdoor surveillance and intruder detection by a mobile robot. The robot must be able to discern humans from other non-human objects, e.g., small animals. More generally, the problem of detecting and localizing human presence has been a widely studied problem due to its potential military, safety, security, and entertainment applications. A number of technologies can be used to detect human signatures and/or track human. However, computer vision has limited ability to detect humans in poor visibility conditions (e.g., at night, haze, fog, rain, and smoke, etc.). Similarly, the performance of infrared detectors varies with the ambient temperature conditions. Human LADAR signatures are often not highly discriminable from other moving clutter, and LADAR performance is degraded in dusty and foggy conditions. UWB radar can provide a complementary technology for detecting and tracking humans, particularly in poor visibility or through-wall conditions, as it is little affected by dust and moisture. While this paper considers the problem of detecting and tracking humans based solely on UWB radar signals, UWB radar technology can profitably joined with other human sensing

This work was not supported by the LIG Nex1 Corporation, Yongin, Korea, and the Agency for Defense Development (ADD), Seoul, Korea.

S. Chang and J. W. Burdick are with the California Institute of Technology, Pasadena, California, USA sanghyun@caltech.edu, jwberobotics.caltech.edu

M. Wolf is with the Jet Propulsion Laboratory, Pasadena, California, USA michael.wolf-131531@jpl.nasa.gov modalities to provide more robust tracking and detection in a wider variety of operating conditions.

Compared with RF, microwave, and mm-wave radar [1], [2], UWB radar provides high-resolution ranging and localization capabilities due to the fine temporal resolution afforded by its wide signal bandwidth [3], [4], [5]. However, the complex multi-path scattering behavior of UWB electromagnetic waves (resulting the multitude of observations per target in each scan) and the highly sensitive response to dynamic human posture may pose additional signal processing and detection/tracking problems. In our previous work, Chang, et. al., developed an algorithm for UWB radar-based human detection in an urban environment populated by other non-human moving objects, such as cars and trucks [6]. With an ad-hoc multi-path clustering method, the human detector had better than $80 \%$ detection probability with $1.58 \%$ false alarm rate in an urban environment The need for better multi-path observation clustering and target tracking techniques motivated the development of an Expectation-Maximization Kalman Filter (EMKF) algorithm for UWB radar-based tracking of a fixed number of humans [7]. Thereafter, the tracking method was extended to handle a variable number of targets, along with clutter and temporary occlusions, resulting in the novel formulation of a multiple hypothesis tracking for clusters (MHTC) procedure, based on earlier work by Wolf [8]. The MHTC method allows us to rigorously organize and select the complex data associations inherent in UWB multi-path scattering from multiple targets [9], [10]. In this paper, we develop a human detection and tracking solution for surveillance application as a combination of the human presence detector and the MHTC procedure, so that the ranges and velocities of targets are estimated and each target track is individually tested for the LR human detector with integrating target feature information over time.

Section II presents the simple UWB radar multi-path signal model that underlies our approach, and shows that waveform time-of-arrival can be interpreted as a point process governed by a Gamma probability distribution. Section III reviews our previous EMKF tracking algorithm for a fixed number of targets as well as our previous MHTC algorithm for tracking a variable number of humans. Section IV presents our proposed human presence detection and tracking algorithm, while Section V presents experimental results to illustrate and validate our approach.

\section{UWB Signal Modeling}

As compared with traditional narrowband radar, the wide bandwith of UWB radar (e.g. a $2 \mathrm{GHz}$ bandwidth centered 

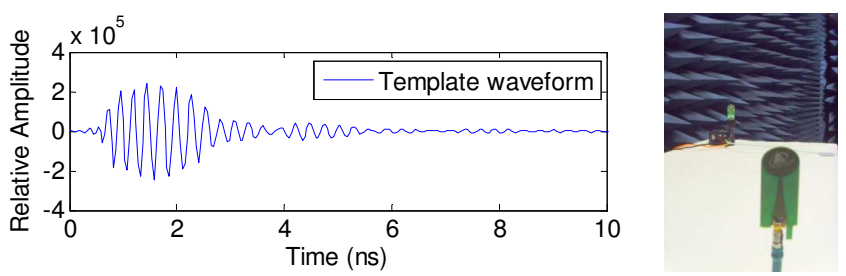

Fig. 1. Measured UWB waveform (left) and measurement setup (right).

at $4 \mathrm{GHz}$ frequency in our experiments) allows for radar waveforms that are highly localized in time. A typical UWB waveform pulse, such as shown in Fig. 1, has a duration of $\sim 0.5-1.5 \mathrm{~ns}$. After the pulse sounds the scattering environment, the returned waveforms are recorded for a fixed interval of time. This interval establishes the effective range of the radar, and the data recorded during one interval is termed a "scan."

\section{A. UWB Scattered Waveform Model}

This paper considers a mono-static UWB radar configuration where waveform pulses are transmitted from a single omni-directional antenna and the scattered waveforms are received by a collocated omni-directional antenna. An effective human detection/tracking strategy requires a model of UWB radar waveform propagation and scattering, e.g., interaction with the human body. A perfectly reflecting target, e.g. a metal plate with an infinite area, returns the impinging UWB electromagnetic wave along a single-path. However, for targets characterized by complex shapes whose spatial extent is larger than the transmitted UWB signal pulse width, e.g. the human body, the returned UWB radar signal consists of multipath components [11], as the impinging UWB electromagnetic wave scatters independently from different human body parts at different times with various amplitudes (depending on the distance to the body part and the size, shape, and composition of the scattering part). Each of these different scattering pathways can be considered one component of the returned UWB radar signal. Thus, the returned UWB radar signal $w(t)$ can be approximated by a specular multipath model [6], [12]:

$$
w(t) \approx \sum_{j} a_{j} p\left(t-n_{j}\right),
$$

with $a_{j}$ and $n_{j}$ respectively representing the amplitude and time-of-arrival (TOA) of the $j^{\text {th }}$ component of the received signal, and $p(t)$ is an elementary waveform shape, e.g., the transmitted radar waveform in free space (see Fig. 1). For example, the waveform is recorded over an interval $t \in$ $\left[t_{0}, t_{\max }\right]$, which corresponds to a range of $r \in\left[r_{0}, r_{\max }\right]=$ $\left[c t_{0} / 2, c t_{\max } / 2\right]$, where $c$ is the speed of light (see details in [6]). The specular multipath model is an approximation whose simplicity allows for real-time processing without compromising UWB radar's high time-resolution capability. Each path's TOA and amplitude can be estimated by the applying the CLEAN algorithm (with a given waveform template) to a scan [6], [13].

\section{B. Human Scattered Waveform Characterization}

In order to understand the basic scattering behavior, we constructed a database of UWB radar scans obtained while a human walked randomly in an open field within the vicinity of the radar (see details in [7]). The radar returns were calibrated and processed using the CLEAN algorithm to extract the amplitudes and TOAs of the scattering multipath components. These returns were then manually segmented to ensure a correct data association between detected scatter paths and the human target. To characterize scattered waveforms from moving humans, we introduce two variables: human range and adjusted time-of-arrival. The human target's nominal range is defined as the first moment of the power range profile $r$ [6]:

$$
r=\frac{\sum_{j \in \Omega} a^{\prime 2} R_{j}}{\sum_{j \in \Omega} a^{\prime 2}},
$$

where $a^{\prime}=a_{j} R_{j}^{2}$ is the $j^{\text {th }}$ scattering path's amplitude normalized at $1 \mathrm{~m}$ (where the free space loss is compensated for the round-trip range), $R_{j}=\left[n_{j} \cdot c\right] / 2$ is the $j^{\text {th }}$ scattering path's range ${ }^{1}, n_{j}$ is the TOA of the $j^{\text {th }}$ scatter component, and $\Omega$ is a set of path indices associated with the human target. It is convenient to introduce an adjusted TOA (ATOA) variable:

$$
\delta_{j}(r)=R_{j}-r+K,
$$

where $r$ is the range to the human, and $K$ is a constant offset related to the radar delay spread of a typical human.

Our studies have found that the ATOA histograms have a behavior consistent with a point process, thus the monostatic UWB radar scattering process for walking humans, under the specular multipath model in Equation (1), can be interpreted as point process governing the ATOAs. After studying common univariate distributions, we found that the ATOA histogram was best fit by a Gamma distribution whose mode lies at the human target location and whose probability density function $(\mathrm{PDF}) f_{\Gamma}(\delta ; \kappa, \theta)$ is:

$$
f_{\Gamma}(\delta ; \kappa, \theta)=\delta^{\kappa-1} \frac{\exp (-\delta / \theta)}{\theta^{\kappa} \Gamma(\kappa)} \text { for } \delta>0,
$$

where $\Gamma(\cdot)$ is the Gamma function, and $\kappa, \theta$ are respectively the Gamma distribution's shape and scale parameters ${ }^{2}$. In our application, the $\kappa$ parameter is a fixed value characteristic of humans, which is estimated from the database at $\kappa=7.60$ in Fig. 2. The $\theta$ parameter is related to target location, and is estimated during the tracking process. While our choice of the Gamma distribution was based on an empirical study, we note that the Gamma distribution exactly models the distribution of arrival times for Poisson distributed events. It is thus a plausible model for multi-path human scatter ATOAs.

\footnotetext{
${ }^{1}$ We refer to TOA and range interchangeably in the paper

${ }^{2}$ All empirical ATOA are adjusted to be positive with $K=0.533 \mathrm{~m}$.
} 


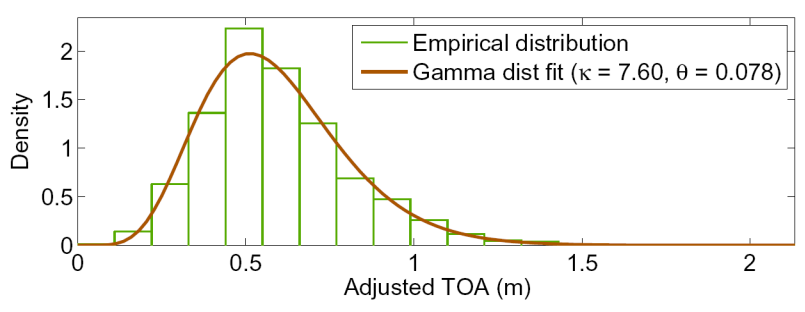

Fig. 2. Histogram of multi-path ATOA, with Gamma distribution fit.

\section{Multiple Human Target Tracking}

For simplicity of exposition, this section summarizes our prior work [7], [9], [10], which forms the basis for the new developments of this paper. First, we show how to track a fixed number of humans using an Expectation-Maximization Kalman Filter (EMKF) algorithm, where the expectationmaximization (EM) algorithm simultaneously associates individual scatter paths to each target and estimates each target's state. Second, we show how to add a multiple target tracking (MTT) capability to this framework via the use of a cluster-based MHT procedure.

\section{A. Tracking a Fixed Number of Human Targets}

We define the state vector $\mathbf{x}$ of a human target as $\mathbf{x}=$ $\left[\begin{array}{ll}r & v\end{array}\right]^{T}$, where $r$ and $v$ respectively denote the range and velocity (time rate of change of the range) of the human target, and $(\cdot)^{T}$ denotes the transpose. For simplicity, we use a simple random walk model to model human dynamics:

$$
\mathbf{x}_{k+1}=\left[\begin{array}{cc}
1 & \Delta T \\
0 & 1
\end{array}\right] \mathbf{x}_{k}+\left[\begin{array}{l}
0 \\
\omega
\end{array}\right]=A \mathbf{x}_{k}+B \omega
$$

where $\omega$ is zero-mean white Gaussian noise with covariance $q^{2}, B=\left[\begin{array}{ll}0 & 1\end{array}\right]^{T}$, and $\Delta T$ is the scan time interval. The covariance of the process noise $B \omega$ is equal to $Q=$ $\operatorname{diag}\left(0, q^{2}\right)$. Note that our algorithm readily incorporates more complicated target dynamic models.

Based on the characterization of human UWB scatter as a point process, we model the multi-target multi-path scattering process as a mixture model, where each mixture component is a Gamma distribution associated with an individual human target. If there are $G$ human targets in the observation environment, where $G$ is known, the likelihood of the $N$ TOA observations $Y^{k}=\left\{n_{j, k}\right\}_{j=1}^{N}$ in the $k^{\text {th }}$ scan, is given by:

$$
p\left(Y^{k} \mid \Theta^{k}\right)=\prod_{j=1}^{N} \sum_{g=1}^{G} \pi_{k}^{g} f_{\Gamma}\left(\delta_{i, k}\left(r_{k}^{g}\right) ; \kappa_{k}^{g}, \theta_{k}^{g}\right),
$$

where $\Theta_{k}$ is the set of model parameters in the $k^{\text {th }}$ scan: $\Theta^{k}=\left\{\pi_{k}^{g}, r_{k}^{g}, \kappa_{k}^{g}, \theta_{k}^{g}\right\}_{g=1}^{G} \cdot{ }^{3}$ Let $\hat{\mathbf{x}}_{k \mid l}^{g}$ and $P_{k \mid l}^{g}$ respectively denote the state estimate of the $g^{\text {th }}$ human target and its error covariance in the $k^{\text {th }}$ scan, given measurements up to time $l$.

An Expectation Maximization Kalman Filter (EMKF) algorithm to update the $G$ target state estimates from the

\footnotetext{
${ }^{3}$ Subscripts refer to time (or scan number) while superscripts are the target index
}

radar scan obtained in the $k^{\text {th }}$ scan is given below. It unites a basic Kalman Filter (KF) state estimator with a data association process (implicitly carried out by the EMalgorithm) that associates individual multi-path returns to specific targets. The path-to-target association probability is modeled as the latent variable of the EM algorithm. Like any tracking algorithm, models for the uncertainties in the dynamic process and the measurements are required for effective tracking. The measurement error covariance used in the algorithm is the sum of a fixed term that describes the inherent noise in the radar processing circuitry, plus a data dependent term that describes the measurement quality and depends upon the number of multi-path returns obtained in a single scan (finite sample effect).

1. Input : TOAs $\left\{n_{j, k}\right\}_{j=1}^{N}$ of the G humans scatter paths at time $k$ (calculated by applying the CLEAN algorithm to scan $k)$.

2. Initialize: Initialize constant parameters: Gamma distribution parameter $\kappa$ (fixed for typical humans), offset $K$ (see footnote 2), dynamic model transition matrix $A$, measurement matrix $H=\left[\begin{array}{ll}1 & 0\end{array}\right]$, process noise $Q$, the constant part of range measurement error covariance $R_{\text {fixed }}$, and iteration threshold $T_{E M}$. Initialize the mixture model parameters $\hat{\Theta}^{k,(0)}$-typically the estimate from $t_{k-1}$ serves as the starting point. Set counter $i=0$.

3. KF Dynamic propagation step : Given the estimate $\hat{\mathbf{x}}_{k-1 \mid k-1}^{g}$ with $P_{k-1 \mid k-1}^{g}$ at time $k-1$, calculate the state estimate $\hat{\mathbf{x}}_{k \mid k-1}^{g}$ and its covariance $P_{k \mid k-1}^{g}$ at time $k$ as

$$
\begin{aligned}
\hat{\mathbf{x}}_{k \mid k-1}^{g} & =A \hat{\mathbf{x}}_{k-1 \mid k-1}^{g}, \\
P_{k \mid k-1}^{g} & =A P_{k-1 \mid k-1}^{g} A^{T}+Q .
\end{aligned}
$$

Set the initial estimate of the human range $\hat{r}_{k}^{g,(0)}=\hat{r}_{k-1}^{g, E M}$. 4. EM algorithm: initialize the EM algorithm (steps 5 and 6) iteration counter, $i=1$.

5. EM E-step : Using the current $i^{\text {th }}$ iteration parameter estimates $\hat{\Theta}^{k,(i)}$ and measurements $Y$, compute the conditional expectation $\hat{z}_{j g}^{(i+1)}=E\left[z_{j g} \mid n_{j, k}, \hat{\Theta}^{k,(i)}\right] \in[0,1]$ as

$$
\hat{z}_{j g}^{(i+1)}=\frac{\pi_{k}^{g} f_{\Gamma}\left(\delta_{j, k}^{g,(i)} ; \kappa, \hat{\theta}_{k}^{g,(i)}\right)}{\sum_{n=1}^{G} \pi_{k}^{n} f_{\Gamma}\left(\delta_{j, k}^{n,(i)} ; \kappa, \hat{\theta}_{k}^{n,(i)}\right)},
$$

where $\hat{z}_{j g}$ is the probability that multi-path component $j$ is generated by target $g$-i.e., the TOA observation to target range measurement association probability.

The ATOAs are calculated by the current $i^{\text {th }}$ iteration human range estimate as

$$
\delta_{j, k}^{g,(i)}=c n_{j, k} / 2-\hat{r}_{k}^{g,(i)}+K .
$$

6. EM M-step : Given $\hat{z}_{j g}^{(i+1)}$, find the parameter estimates $\hat{\Theta}^{k,(i)}$ that maximizes the complete-data log-likelihood function:

$$
l_{C D}\left(\Theta^{k} \mid Y^{k}, Z\right)=\sum_{j=1}^{N} \sum_{g=1}^{G} z_{j g} \log \left[\pi_{k}^{g} f_{\Gamma}\left(\delta_{j, k}^{g} ; \kappa, \theta_{k}^{g}\right)\right],
$$


which models the probability, given the set of all data association assignments $Z=\left\{z_{j g}\right\}$, that the multi-path range measurements are generated by $G$ targets located at positions parameterized by $\Theta^{k}$. Using the Lagrange multiplier method with the constraint of $\pi_{k}^{g} \geq 0$ and $\sum_{g=1}^{G} \pi_{k}^{g}=1$, one obtains the following estimates of $\pi_{k}^{g}$ and $\theta_{k}^{g}$ :

$\hat{\pi}_{k}^{g,(i+1)}=\frac{\sum_{j=1}^{N} \hat{z}_{j g}^{(i+1)}}{N}$ and $\hat{\theta}_{k}^{g,(i+1)}=\frac{\sum_{j=1}^{N} \hat{z}_{j g}^{(i+1)} \delta_{j, k}^{g,(i)}}{\kappa \sum_{j=1}^{N} \hat{z}_{j g}^{(i+1)}}$.

The range estimate at the mode of the distribution is:

$$
\hat{r}_{k}^{g,(i+1)}=(\kappa-1) \hat{\theta}_{k}^{g,(i+1)}+\hat{r}_{k}^{g,(i)}-K .
$$

7. Iteration criterion: If $\sum_{g=1}^{G}\left|\hat{r}_{k}^{g,(i+1)}-\hat{r}_{k}^{g,(i)}\right|>T_{E M}$, go to step 5 with $i \leftarrow i+1$. Otherwise, each TOA $n_{j, k}$ is assigned to the $g^{* \text { th }}$ human via

$$
g^{*}=\arg \max _{g} \hat{z}_{j g} .
$$

Set the estimate of human range $\hat{r}_{k}^{g, E M}=\hat{r}_{k}^{g,(i+1)}$, and the estimation error variance $R_{k}=R_{f i x}+\left[(\kappa-1) \hat{\theta}_{k}^{g,(i+1)}\right]^{2} / \kappa N$.

8. KF Measurement Update : Set the human range measurement $y_{k}^{g}=\hat{r}_{k}^{g, E M}$. Update the Kalman gain $K_{k}^{g}$, the a posterior state estimate $\hat{\mathbf{x}}_{k \mid k}^{g}$, and the error covariance $P_{k \mid k}^{g}$ as

$$
\begin{aligned}
K_{k}^{g} & =P_{k \mid k-1}^{g} H^{T}\left(H P_{k \mid k-1}^{g} H^{T}+R_{k}\right)^{-1}, \\
\hat{\mathbf{x}}_{k \mid k}^{g} & =\hat{\mathbf{x}}_{k \mid k-1}^{g}+K_{k}^{g}\left(y_{k}^{g}-H \hat{\mathbf{x}}_{k \mid k-1}^{g}\right), \\
P_{k \mid k}^{g} & =\left(I-K_{k}^{g} H\right) P_{k \mid k-1}^{g} .
\end{aligned}
$$

Experimental validation of this method for the case of a fixed number of humans can be found in [7].

\section{B. Tracking a Variable Number of Human Targets}

In a realistic environment, the number of human targets will vary with time, as targets may go in and/or out of the observation volume. Additionally, the tracking system should also be able to handle clutter (e.g. non-human objects and false measurements) and missed detections (temporary occlusions), allowing for an appropriate segmentation process, simultaneously. The algorithm summarized above also has no inherent mechanism to construct consistent tracks across multiple scans. Thus, it is necessary to develop a MultiTarget-Tracking (MTT) solution for these practical realities. In this problem, the MTT technique should solve two types of data association problems: all multipath scatter components must first be segregated according to their generating source (the observation-measurement association problem or the multipath scatter-cluster association problem); and then each scattering cluster must be associated to clusters from previous scans, thus tracking the UWB scattering response of putative human targets (the measurement-target or track assignment association problem). This differs from the standard MTT problem which only focuses on the single track assignment data association problem.

We propose to use a Multi-Hypothesis-Testing (MHT) approach which maintains many possible data association

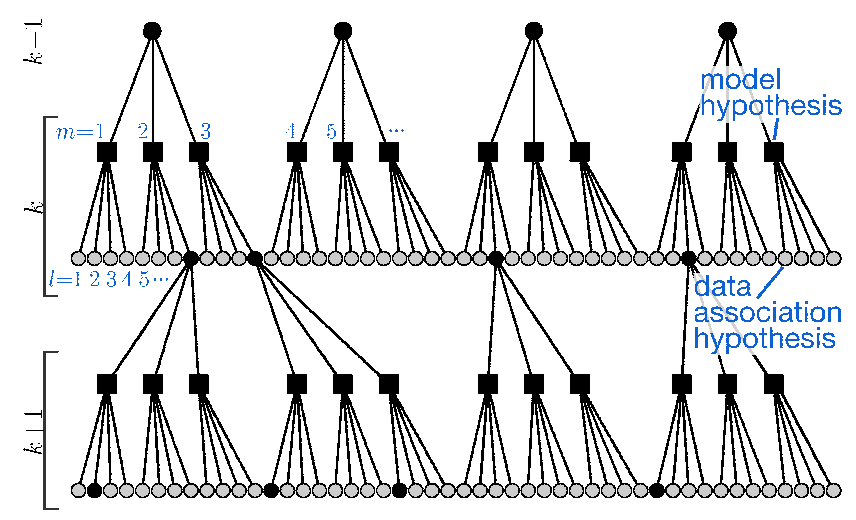

Fig. 3. MHTC hypothesis tree structure, illustrating the integration of model hypotheses into the traditional MHT framework. Squares represent model hypotheses (i.e., clustering output) and black circles represent surviving data association hypotheses at each time step.

hypotheses and propagates the corresponding target state estimates for each hypothesis, implicitly deferring decisions if necessary in anticipation that subsequent data measurements will resolve any ambiguity [14]. However, unlike traditional MHT in military radar and computer vision tracking applications [15], [16], this problem has the additional complexity that targets are only sensed indirectly via clusters of scattering path observations. To incorporate this additional complexity, we adapt a recently developed MHTC method [8] that was originally developed for dynamic sorting and tracking of neural signals. This algorithm propagates various possibilities for how to assign observations to clusters and then clusters to existing target tracks. It uses a delayed decision-making logic to resolve data association or track association ambiguities. It also maintains several options, termed model hypotheses, for how to cluster the observations in each scan (see Fig. 3 and 4). This combination of clustering and tracking in a single solution enables the MHTC to robustly maintain the identities of cluster-producing targets. See [8], [9], [10] for details.

\section{Human Detection-And-Tracking Algorithm}

In this section, a human presence detection-and-tracking algorithm is presented as a solution for surveillance application. The algorithm is proposed in Fig. 5, describing a single algorithm cycle for each updated radar scan. First, a moving target indication (MTI) system is applied to each incoming radar scan for the following reason. Since UWB radar scatters from both stationary and moving objects, all scatters obtained from a complex test environment must be analyzed for human target candidates (even including highly human-unlike scatters). To reduce the high computational cost associated to such analysis, an MTI system, summarized in [6], is used to eliminate highly human-unlike scatters. Second, the CLEAN algorithm [13] is applied to the MTI response of radar scan to obtain estimated TOAs and amplitudes of the decomposed multipath components. Third, the MHTC procedure in Section III is applied to output of the CLEAN algorithm. Fourthly, for each target track, 


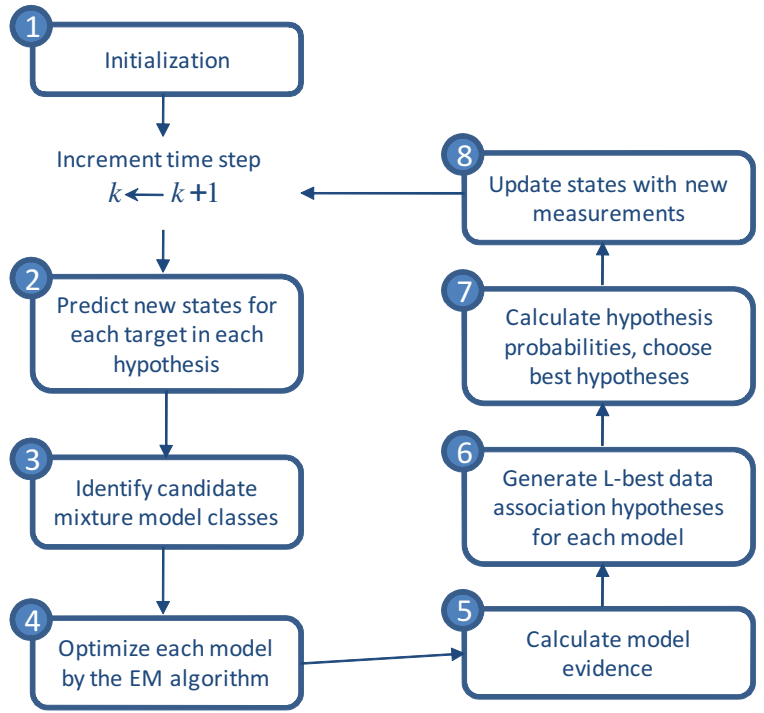

Fig. 4. MHTC process diagram.

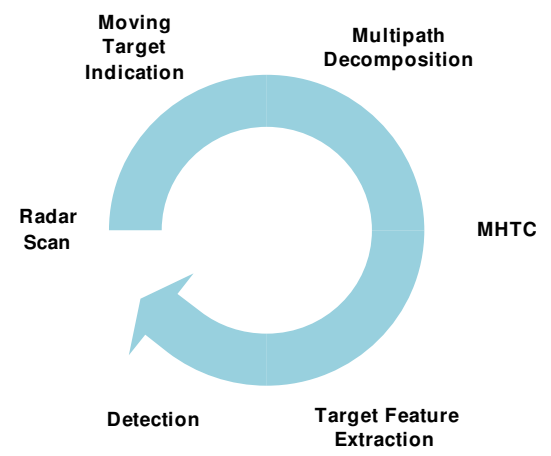

Fig. 5. An algorithm cycle of human presence detection and tracking framework.

the features of maximum path magnitude and RMS range spread are estimated. Finally, a hypothesis testing process (or detection process) determines whether the tested track is interpreted/detected as a human or not. The following subsections will describe the target feature extraction technique in detail, as well as the detection process.

\section{A. Feature Extraction}

The specular multipath model Equation (1) is a computationally useful signal representation that reduces UWB waveform representation to 2-dimension (path amplitude and TOA). To differentiate human scatter from non-human scatter, discriminatory target features are required. Two features are proposed for discriminating human and non-human scatter under the specular multipath model: (1) the path's maximum magnitude, which is relevant to target composition and cross-section size; and (2) the RMS delay spread of multipath delay profile (or the RMS range spread), which is relevant to effective scattering point spatial extent in range/depth.

From Equation (1), the path's maximum magnitude can be represented as

$$
a_{\max }=\max _{j \in \Omega}\left|a_{j} R_{j}^{2}\right|,
$$

where $\Omega$ is the set of path indices associated with the target, and $R_{j}^{2}=\left(c n_{j} / 2\right)^{2}$ is normalization factor. For simplicity of exposition, the first moment of the power range profile is defined as a function of normalized path amplitude by $\bar{R}=\frac{\sum_{j \in \Omega} R_{j}\left(a_{j} R_{j}^{2}\right)^{2}}{\sum_{j \in \Omega}\left(a_{j} R_{j}^{2}\right)^{2}}$. Then, the RMS range spread $R_{r m s}$ can be calculated as

$$
R_{r m s}=\left[\frac{\sum_{j \in \Omega}\left(R_{j}-\bar{R}\right)^{2}\left(a_{j} R_{j}^{2}\right)^{2}}{\sum_{j \in \Omega}\left(a_{j} R_{j}^{2}\right)^{2}}\right]^{\frac{1}{2}} .
$$

\section{B. Detection}

The detection process on each target can be viewed as a binary hypothesis test over the extracted target feature $\Theta=\left\{a_{\max }, R_{r m s}\right\}$ of the following null hypothesis, $\mathcal{H}_{0}$, and the alternative hypothesis, $\mathcal{H}_{1}$ :

$$
\begin{aligned}
& \mathcal{H}_{0}: \text { the target is non-human } \\
& \mathcal{H}_{1}: \text { the target is human. }
\end{aligned}
$$

The hypotheses are evaluated using a likelihood ratio test (LRT) $\Lambda(\Theta)$ :

$$
\Lambda(\Theta)=\frac{\mathcal{L}\left(\Theta \mid \mathcal{H}_{1}\right)}{\mathcal{L}\left(\Theta \mid \mathcal{H}_{0}\right)}=\frac{p\left(\Theta \mid \mathcal{H}_{1}\right)}{p\left(\Theta \mid \mathcal{H}_{0}\right)}
$$

where the likelihood function $\mathcal{L}\left(\Theta \mid \mathcal{H}_{i}\right)$ given target feature $\Theta=\left\{a_{\max }, R_{r m s}\right\}$ under the hypothesis $\mathcal{H}_{i}$ is defined by the conditional probability distribution $p\left(\Theta \mid \mathcal{H}_{i}\right)$ of two features under the hypothesis $\mathcal{H}_{i}$, for $i=0,1$. Provided that $a_{\text {max }}$ and $R_{r m s}$ are all independent, the LRT has the form:

$$
\Lambda(\Theta)=\frac{p\left(a_{\max } \mid \mathcal{H}_{1}\right) p\left(R_{r m s} \mid \mathcal{H}_{1}\right)}{p\left(a_{\max } \mid \mathcal{H}_{0}\right) p\left(R_{r m s} \mid \mathcal{H}_{0}\right)} \underset{\mathcal{H}_{1}}{\stackrel{\mathcal{H}_{0}}{\lessgtr}} T_{D}
$$

where the detection threshold $T_{D}$ determines the performance of the detection process.

The LRT detector can determine whether the tested target track is detected as a human or not in every single scan. While this detector can be processed quickly with low latency, it does not utilize mutual information or dynamics across successive scans. To integrate information over time, we use a voting method that calculates number of times that the target is detected as a human over the last $N_{v}$ LRT detector cycles. Human presence is declared when greater than $50 \%$ of the $N_{v}$ scans vote positively for human presence.

\section{EXPERIMENTAL RESULTS}

To test the human detection and tracking algorithm, UWB mono-static radar measurements were conducted in an open field by using a Time Domain PulsOn 210 mono-static UWB radar for time-varying number of targets: human targets walking in and out of the radar observation volume and an aluminum foil covered basketball as a representative substitution of small animal. Fig. 6(a) shows 800 unprocessed scans (each column represents the magnitude of a single scanned 


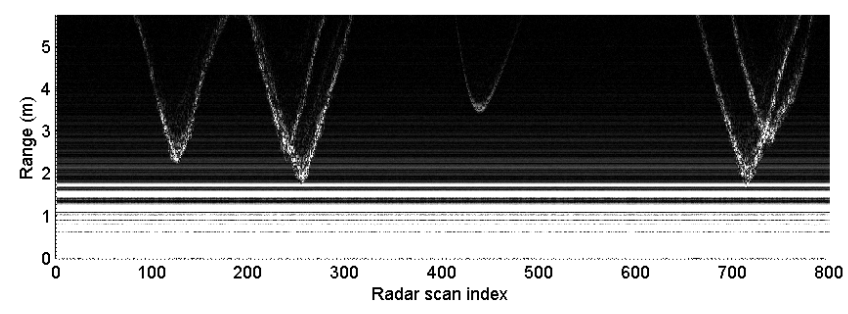

(a) Representation of 800 unprocessed scans.

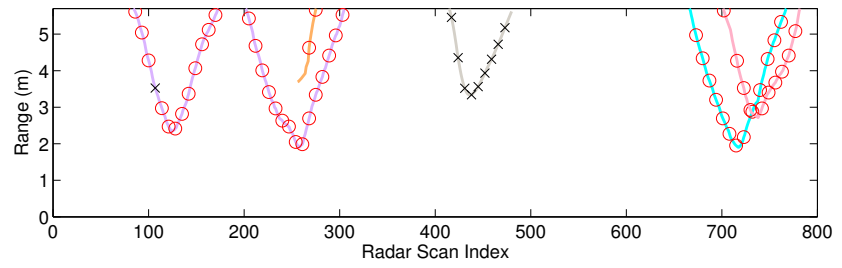

(b) Estimated 5-target ranges and human detection results (o: human, X: non-human).

Fig. 6. The human detection and tracking algorithm results.

waveform over the sensing range, where the waveform magnitude is scaled from 0 in black to 7000 in white. Magnitudes over 7000 are clipped to 7000). Moving targets' trajectories can be seen in Fig. 6(a), while the horizontal patterns in the near range $(<2.2 \mathrm{~m})$ represent direct antenna coupling effects. During $\sim 63$ seconds of recording time, 5 number of targets had been in the radar range over the following radar scan index: (1) human\#1: $81 \sim 295$; (2) human\#2: 188 265; (3) basketball: 413 476; (4) human\#4: 662 767; and (5) human $\#$ 5: $699 \sim 769$. The radar scanning period $\Delta T$ was $0.0786 \mathrm{sec} / \mathrm{scan}=(12.7 \mathrm{scans} / \mathrm{sec})^{-1}$, and the waveform sampling resolution was 41.33 ps with the range resolution of $0.0062 \mathrm{~m}$.

The MTI output of the radar returns was processed using the CLEAN algorithm with $T_{\text {clean }}=5 \times 10^{4}$ to estimate the amplitudes and TOAs of the scattering components. These measurements were then processed using the MHTC algorithm with parameters of $\kappa=7.60, K=0.533 \mathrm{~m}, L=$ $6, \mathrm{P}_{\mathrm{d}, j}=0.98, \lambda_{\nu}=0.01$, and $\lambda_{\phi}=0.0105$. The detection process was conducted with the extracted target features ${ }^{4}$, where $N_{v}=7$. Fig. $6(\mathrm{~b})$ shows that human and non-human targets are tracked for a variable number of human even with the crossing of two tracks in scan 732 (except the case when human ${ }^{\#}$ track was merged to human ${ }^{\#} 1$ track from scan 188 to 247 . Human detection results were correct except only one case at around scan 100.

\footnotetext{
${ }^{4}$ The conditional distributions in (9) are empirically constructed [6] and represented as follows:

$$
\begin{aligned}
p\left(a_{\max } \mid \mathcal{H}_{1}\right) & =f_{\mathcal{N}}\left(x ; 104.6,3.70^{2}\right), \\
p\left(a_{\max } \mid \mathcal{H}_{0}\right) & =\frac{1}{12}, \text { for } a_{\max } \in[93,105], \\
p\left(R_{r m s} \mid \mathcal{H}_{1}\right) & =f_{\mathcal{N}}\left(x ; 0.1157,0.0316^{2}\right), \\
p\left(R_{\text {rms }} \mid \mathcal{H}_{0}\right) & =f_{\mathcal{N}}\left(x ; 0.0303,0.0120^{2}\right),
\end{aligned}
$$
}

where normal distribution with the mean $m$ and the variance $\sigma^{2}$ is denoted by $f_{\mathcal{N}}\left(x ; m, \sigma^{2}\right)$ on the domain $x \in(-\infty, \infty)$.

\section{CONCLUSIONS}

Ultra-Wide-Band radar offers a complementary technology for detection and tracking humans, as it works well in conditions (such as in the dark, or in dusty, foggy, rainy environments) where the performance of other sensing modalities degrades. However, the different nature of the UWB signal requires new processing and tracking algorithms. In this paper we developed a human detection and tracking solution as a combination of a human presence detector and the MHTC procedure. Ongoing work seeks to improve our method to identify clutter in the UWB return signal, to apply array signal processing or synthetic aperture radar (SAR) technique for 2-dimensional localization and/or mobile platform application and to extend the approach to multi-antenna configurations as well as LADAR-and-radar or vision-and-radar multi-modal human detection and tracking techniques.

\section{REFERENCES}

[1] M. I. Skolnik, Radar Handbook, New York: McGraw Hill, 1970.

[2] P. Z. Peebles, Jr., Radar Principles, New York: Wiley, 1998.

[3] R. M. Narayanan, "Through-Wall Radar Imaging using UWB Noise Waveforms," J. Franklin Institute vol. 345, no. 6, pp. 659-678, Sept. 2008.

[4] C. Chang, and A. Sahai, "Object tracking in a 2D UWB sensor network," Proc. $38^{\text {th }}$ Asilomar Conf. on Signals, Systems and Computers, vol. 1, pp. 1252-1256, 2004.

[5] R. A. Scholtz, D. M. Pozar, and W. Namgoong, "Ultra-Wideband Radio, EURASIP Journal of Applied Signal Processing, No. 3, 2005, pp. $252-272$.

[6] S. Chang, N. Mitsumoto, J.W. Burdick, "An algorithm for UWB radarbased human detection," in Proc. IEEE RadarCon 2009, Pasadena, CA, May 4-8, 2009.

[7] S. Chang, R. Sharan, M. Wolf, N. Mitsumoto, and J.W. Burdick, "UWB radar-based tracking of humans," in Proc. IEEE RadarCon 2009, Pasadena, CA, May 4-8, 2009.

[8] M. Wolf, "Target Tracking Using Clustered Measurements, with Applications to Autonomous Brain-Machine Interfaces," Ph.D. thesis, Department of Mechanical Engineering, California Institute of Technology, Pasadena, CA, June 2008.

[9] S. Chang, M. Wolf, and J. W. Burdick, "An MHT algorithm for UWB radar-based multiple human target tracking," in Proc. IEEE ICUWB, Vancouver, Canada, September, 2009.

[10] S. Chang, R. Sharan, M. Wolf, N. Mitsumoto, and J. W. Burdick, "Prople Tracking with UWB Radar Using a Multiple-Hypothesis Tracking of Clusters (MHTC) Method," Springer International Journal of Social Robotics (Special issue on people detection and tracking), DOI:10.1007/s12369-009-0039-x, 2010.

[11] E. F. Knott, J. F. Shaeffer, and M. T. Tuley, Radar Cross Section, $2^{\text {nd }}$ ed., Artech House, Norwood, MA, 1993.

[12] H. Hashemi, "Impulse response modeling of the indoor radio propagation channels," IEEE Trans. Select. Areas Commun., vol. 11, no. 7, Sep. 1993.

[13] S. M. Yano, "Investigating the ultra-wideband indoor wireless channel," in Proc. IEEE VTC Spring Conf., vol. 3, pp. 1200.1204, 2002.

[14] D. B. Reid, "An algorithm for tracking multiple targets," IEEE Transactions on Automatic Control, 24(6):843-854, December 1979.

[15] S. S. Blackman and R. Popoli, Design and Analysis of Modern Tracking Systems, Artech House, Norwood, MA, 1999.

[16] G. W. Pulford, "Taxonomy of multiple target tracking methods," IEE Proceedings - Radar, Sonar \& Navigation, 152(5):291 304, 2005. 